\title{
QUALITATIVE REVIEW OF ANTIBIOTIC USE FOR NEONATAL SEPSIS
}

\author{
TJIO IE WEI ${ }^{1}$, PURWANTYASTUTI ASCOBAT ${ }^{1}{ }^{1}$, RINAWATI ROHSISWATMO ${ }^{2}$, INSTI INSTIATY ${ }^{1 *}$
}

${ }^{1}$ Department of Pharmacology and Therapeutics, Faculty of Medicine, Universitas Indonesia, Jakarta, 10430, Indonesia. ${ }^{2}$ Department of Child Health, Faculty of Medicine, Universitas Indonesia, Jakarta, 10430, Indonesia. Email: instiaty@yahoo.com

Received: 06 August 2018, Revised and Accepted: November 2018

ABSTRACT

Objective: The aim of this study is to evaluate the antibiotic use in neonates with sepsis.

Methods: An observational retrospective study was conducted using medical records of neonates diagnosed with early-/late-onset sepsis who were prescribed antibiotics and who were treated in the neonatal intensive care unit (NICU) at the Dr. Cipto Mangunkusumo Hospital between January 1 and December 31, 2015. Patient records were screened for antibiotic use; qualitative analyses were performed using the Gyssens algorithm. Concordance of empirical antibiotic prescriptions with subsequent blood culture and sensitivity tests was evaluated.

Results: A total of 176 sepsis cases included 80 and 96 neonates with normal and low birth weights (LBWs), respectively. Ampicillinsulbactam+gentamycin, which is indicated in local guidelines as the first-line antibiotic combination for neonatal sepsis, was most frequently prescribed. In the normal birth weight group, appropriate antibiotic use (Gyssens Category I) was found in $89.7 \%$ of cases, whereas Gyssens Category V (no indication) was found in $4.54 \%$ of cases. In the LBW group, $88.1 \%$ and $6.2 \%$ of cases were included in Gyssens Categories I and V, respectively. Only $17.5 \%$ and $13.5 \%$ cultured blood specimens from normal and LBW groups, respectively, yielded positive results; the most commonly identified bacteria were Acinetobacter baumannii and Klebsiella pneumonia. All isolates were resistant to ampicillin-sulbactam; only $7.4 \%$ were sensitive to gentamicin.

Conclusion: Antibiotic use for neonatal sepsis in NICU in this study can be considered appropriate, suggesting proper implementation of antimicrobial guidelines. However, high rates of resistance to the first-line antibiotics for neonatal sepsis are concerning.

Keywords: Antibiotic, Gyssens, Neonatal sepsis.

(C) 2018 The Authors. Published by Innovare Academic Sciences Pvt Ltd. This is an open access article under the CC BY license (http://creativecommons. org/licenses/by/4. 0/) DOI: http://dx.doi.org/10.22159/ijap.2018.v10s1.80

\section{INTRODUCTION}

Neonatal sepsis follows invasive infection, usually bacterial, that occurs during the neonatal period. In a report from the WHO, the case fatality rate of neonatal sepsis was estimated at approximately $40 \%[1,2]$. Although antibiotic treatments should be initiated immediately on suspicion of neonatal sepsis [3-6], in 20-30\% of neonatal cases of sepsis, antibiotics are unnecessarily prescribed and may increase morbidity, health-care costs, and resistance to antimicrobials. Hence, to ensure appropriate prescriptions, such prescriptions should be monitored and evaluated regularly. To this end, an antimicrobial resistance control program was implemented at the Dr. Cipto Mangunkusumo Hospital (RSCM) Jakarta, in 2009, although the appropriateness of antibiotic use in neonates with sepsis has not been assessed in the neonatal intensive care unit (NICU). Herein, we performed qualitative analyses of antibiotic use in neonates with sepsis who were treated in the NICU at RSCM.

\section{METHODS}

An observational retrospective study was performed using the records of neonates with sepsis who were treated in the NICU at RSCM between January 1 and December 31, 2015. The included neonates were diagnosed with clinical sepsis or had abnormal sepsis marker levels that were indicative of early-onset neonatal sepsis (EONS) appearing in the first $72 \mathrm{~h}$ after birth or late-onset neonatal sepsis (LONS) appearing at $\geq 72 \mathrm{~h}$ after birth, and were treated with antibiotics [3]. Neonates with birth weights of $<1500 \mathrm{~g}$ were excluded from the study and the diagnoses in medical records were considered accurate. This study was approved by the Ethics Committee of the Faculty of Medicine Universitas Indonesia (No. 733/UN2.F1/ETIK/2016). Gestational ages, birth weights, details of labor (spontaneous, cesarean section), types of sepsis (EONS or LONS), types, frequencies, doses, and routes of antibiotic administration and results of culture, and sensitivity tests were included in the analyses.

Qualitative evaluations of antibiotic use were performed using the Gyssens algorithm, through which antibiotic use is divided into the following six categories: Category I, appropriate use; Categories II a, II b, or II c, inappropriate dose, dosing interval, or route of administration, respectively; Categories III a or III b, inappropriately long or short duration of treatment, respectively; Categories IV a, IV b, IV c, or IV d, more effective, less toxic, less expensive, or narrower spectrum of available antibiotics, respectively; Category V, no indication for antibiotics, and Category VI, antibiotic use not supported by sufficient data.

The appropriateness of the antibiotic use was determined with reference to the Pediatric Department Guidelines of Clinical Practice at RSCM, the Royal Women's Hospital Neonatal Pharmacopoeia, the Drug Dose by Shann, the profile of bacterial and antibiotic susceptibility at RSCM, and other relevant references [7-9]. A pediatric neonatologist was also consulted during evaluations.

\section{RESULTS AND DISCUSSION}

A total of 176 sepsis cases included 96 neonates with low birth weight (LBW) (LBW, <2500 g) and 80 with normal birth weight (NBW) (NBW, $\geq 2.500 \mathrm{~g}$ ), and total numbers of antibiotic prescriptions were 925 for the NBW group and 1505 for the LBW group. Table 1 shows profiles of neonates with sepsis treated in the NICU at RSCM in 2015.

The most commonly administered antibiotic preparation in the NBW (400 prescriptions) and LBW groups (524 prescriptions) was 
ampicillin-sulbactam+gentamycin, which is the first-line empirical antibiotic for neonatal sepsis according to the 2015 guidelines for antibiotic use in the Pediatric Department at RSCM. The second most widely prescribed antibiotic preparation was piperacillintazobactam+amikacin, with 219 prescriptions for the NBW and 462 prescriptions for the LBW groups. This preparation is listed in the guidelines for antibiotic use in the Pediatric Department, RSCM, as the second-line empirical antibiotic treatment for neonatal sepsis, followed

Table 1: Profile of neonates with sepsis in the NICU at RSCM during 2015

\begin{tabular}{|c|c|c|c|}
\hline \multirow[t]{2}{*}{ No. } & \multirow[t]{2}{*}{ Characteristic } & NBW & \multirow{2}{*}{$\begin{array}{l}\text { LBW } \\
\text { n (\%) }\end{array}$} \\
\hline & & n (\%) & \\
\hline \multirow[t]{3}{*}{1} & Sepsis neonates & & \\
\hline & EONS & $78(81.25)$ & $84(87.5)$ \\
\hline & LONS & $18(18.75)$ & $12(12.5)$ \\
\hline \multirow[t]{3}{*}{2} & Gestational age (weeks) & & \\
\hline & $<37$ & $18(18.75)$ & $93(96.87)$ \\
\hline & $\geq 37$ & $78(81.25)$ & $3(3.13)$ \\
\hline \multirow[t]{3}{*}{3} & History of parturient & & \\
\hline & Section cesarian & $60(62.5)$ & $57(59.38)$ \\
\hline & Spontaneous & $36(37.5)$ & $39(40.62)$ \\
\hline \multirow[t]{3}{*}{4} & Outcome & & \\
\hline & Recover & $91(94.79)$ & $80(83.33)$ \\
\hline & Death & $5(5.21)$ & $16(16.67)$ \\
\hline
\end{tabular}

EONS: Early-onset neonatal sepsis, LONS: Late-onset neonatal sepsis,

NBW: Normal birth weight, LBW: Low birth weight

Table 2: Antibiotics prescribed for neonates with sepsis in the NICU at RSCM during 2015

\begin{tabular}{llll}
\hline No. & Antibiotics & \multicolumn{2}{l}{$\begin{array}{l}\text { Number of } \\
\text { prescriptions (\%) }\end{array}$} \\
\cline { 3 - 4 } & & NBW (n=80) & LBW (n=96) \\
\hline 1. & Ampicillin-sulbactam+gentamicin & $400(43.2)$ & $524(34.8)$ \\
2. & Piperacillin-tazobactam+amikacin & $219(23.7)$ & $462(30.7)$ \\
3. & Meropenem & $124(13.4)$ & $193(12.8)$ \\
4. & Cefepime & $91(9.8)$ & $71(4.7)$ \\
5. & Cefoperazone & $45(4.9)$ & $30(2.0)$ \\
6. & Imipenem & $32(3.5)$ & $47(3.1)$ \\
7. & Cefoperazone-sulbactam & $14(1.5)$ & $6(0.4)$ \\
8. & Ampicillin-sulbactam & - & $7(0.5)$ \\
9. & Gentamicin & - & $31(2.1)$ \\
10. & Amikacin & & $54(3.6)$ \\
11. & Metronidazole & - & $30(2.0)$ \\
12. & Tigecycline & - & $35(2.3)$ \\
13. & Levofloxacin & - & $15(1.0)$ \\
& Total & 925 & 1505 \\
\hline
\end{tabular}

NBW: Normal birth weight, LBW: Low birth weight, $\mathrm{n}$ : Number of patients by meropenem, for which 124 and 193 prescriptions were recorded for the NBW and LBW groups, respectively. Table 2 lists antibiotics prescriptions for neonates with sepsis in the NICU at RSCM during 2015.

Qualitative evaluations of antibiotic use were performed using the Gyssens algorithm and are presented in Tables 3 and 4. In the NBW group, antibiotic usage was categorized appropriate (Category I) in 830 cases $(90.4 \%)$, and 2 cases $(0.2 \%)$ were placed in Category III A, $25(2.7 \%)$ in Category IV D, $6(0.7 \%)$ in Category IV C, 13 (1.4\%) in Category IV A, and 42 (4.6\%) in Category V (no indication to use antibiotics; Table 3).

In the LBW group, 1326 of 1505 antibiotic prescriptions (88.1\%) were categorized as appropriate (Gyssens Category I), and $8(0.5 \%)$ were placed in Category III A, 61 (4.1\%) in Category IV B, 17 (1.1) in Category IV D, and $93(6.2 \%)$ in Category V (no indication for antibiotics; Table 4).

Blood cultures were performed for all the NBW and LBW neonates with sepsis before antibiotic administration and proportions of bacterial cultures that yielded positive results were relatively low. In the LBW group, 13 of the 96 patients had positive bacterial cultures (13.5\%), whereas 14 of the 80 patients $(17.5 \%)$ in the NBW group had positive results. Overall, the most common bacteria were Acinetobacter baumannii and Klebsiella pneumonia (each 18.5\%), Escherichia coli and Serratia marcescens (each 14.5\%), and Staphylococcus epidermidis (11.1\%). Of the 27 isolates, all were resistant to ampicillin-sulbactam and $7.4 \%$ only were sensitive to gentamicin, $11.1 \%$ only were sensitive to piperacillin-tazobactam, $14.8 \%$ only were sensitive to amikacin, and $25.9 \%$ only were sensitive to meropenem.

\section{DISCUSSION}

According to the local 2015 guidelines for clinical practice in the Department of Pediatrics at RSCM, the first-line, second-line, and third-line antibiotics for the treatment of neonatal sepsis were ampicillin-sulbactam+gentamicin, piperacillin-tazobactam+amikacin, and meropenem, respectively [7]. In the present study, the three most frequently used antibiotics for neonates with sepsis were in strict accordance with the first-, second-, and third-line antibiotics listed for those conditions in the local guidelines, implying that the guidelines were well implemented.

Antibiotic use in the neonates with sepsis was appropriate (Gyssens Category 1) in almost $90 \%$ of the cases, although antibiotics were prescribed for some patients without indications (Gyssens Category V). In these cases, the antibiotics were prescribed following the failure of empirical antibiotics to improve clinical symptoms, despite negative bacterial cultures.

This is the first study of antibiotic use specifically in neonatal sepsis patients at the RSCM. Satari et al. qualitatively evaluated antibiotic use in the pediatric ward at the RSCM during 2009 and showed that antibiotics were used appropriately in $39.6 \%$ of cases only [10]. In the incipient antibiotic use study by Pamela, appropriate antibiotic use was

Table 3: Antibiotic use for neonatal sepsis in the NBW group based on Gyssens categories

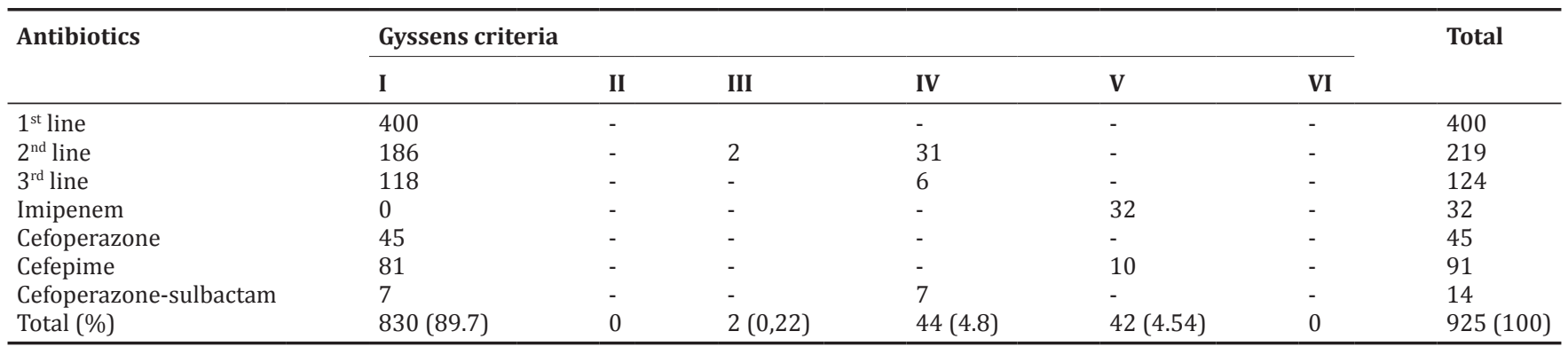

$1^{\text {st }}$ line, ampicillin-sulbactam+gentamycin; $2^{\text {nd }}$ line, piperacillin-tazobactam+amikacin, $3^{\text {rd }}$ line, meropenem, NBW: Normal birth weight 
Table 4: Antibiotic use for neonatal sepsis in the LBW group based on Gyssens categories

\begin{tabular}{|c|c|c|c|c|c|c|c|}
\hline \multirow[t]{2}{*}{ Antibiotics } & \multicolumn{6}{|c|}{ Gyssens category } & \multirow[t]{2}{*}{ Total } \\
\hline & I & II & III & IV & $\mathbf{V}$ & VI & \\
\hline $1^{\text {st }}$ line & 524 & - & - & - & - & - & 524 \\
\hline $3^{\text {rd }}$ line & 191 & - & 2 & - & - & - & 193 \\
\hline Imipenem & - & - & - & 11 & 36 & - & 47 \\
\hline Cefoperazone & 28 & - & - & - & 2 & - & 30 \\
\hline Cefepime & 62 & - & - & - & 9 & - & 71 \\
\hline Cefoperazone-sulbactam & 6 & - & - & - & - & - & 6 \\
\hline Amikacin & 54 & - & - & - & - & - & 54 \\
\hline Gentamicin & 31 & - & - & - & - & - & 31 \\
\hline Ampicillin-sulbactam & 7 & - & - & - & - & - & 7 \\
\hline Levofloxacin & - & - & - & 15 & - & - & 15 \\
\hline Tigecycline & - & - & - & 35 & - & - & 35 \\
\hline Metronidazole & - & - & - & - & 30 & - & 30 \\
\hline Total (\%) & $1326(88.1)$ & 0 & $8(0.5)$ & $78(5.2)$ & $93(6.2)$ & 0 & $1505(100)$ \\
\hline
\end{tabular}

$1^{\text {st }}$ line, ampicillin-sulbactam+gentamycin; $2^{\text {nd }}$ line, piperacillin-tazobactam+amikacin; $3^{\text {rd }}$ line, meropenem, LBW: Low birth weight

recorded in $60.4 \%$ of pediatric patients in the pediatric ward at the RSCM [11]. Encouragingly, the present rate of appropriate antibiotic was almost 90\%, suggesting continued improvement in our department, although the subjects of this study were limited to neonates with sepsis.

Of great concern is all the isolates were resistant to ampicillinsulbactam, which is, in combination with gentamicin, classified as the first-line antibiotic treatment for neonatal sepsis. Resistance to the second- (piperacillin-tazobactam+amikacin) and third-line antibiotics (meropenem) was also high. However, in this study, microbial cultures yielded positive results in $17.5 \%$ only of the specimens from the NBW group and in $13.5 \%$ only of the specimens from the LBW group. These data indicate the necessity of improvements in the success rates of microbial cultures to increase the accuracy of antimicrobial sensitivity assessments.

\section{CONCLUSION}

Antibiotic use for neonatal sepsis in the NICU at the RSCM during 2015 was predominantly appropriate, reflecting good adherence to the guidelines for antimicrobial use. However, the results of bacterial cultures and sensitivity tests indicate the necessity of the regular evaluations of empirical antibiotic regimens for neonatal sepsis.

\section{CONFLICTS OF INTEREST}

All authors declare that they have no conflicts of interest.

\section{REFERENCES}

1. Kardana IM. The pattern of germs and the sensitivity of antibiotics in the perinatology room. Sari Pediatr 2011;12:381-5.

2. Putra PJ. Incidents and factors associated with neonate sepsis at Sanglah hospital Denpasar. Sari Pediatr 2012;14:205-10.

3. Lever A, Mackenzie I. Sepsis: Definition, epidemiology, and diagnosis. BMJ 2007;335:879-83.

4. Nia SS, Modaresahmadi S, Thirumoorthi V, Anwar Q, Prasad S. Multidrug-resistant polymicrobial bacteremia and sepsis: A rare case report. Asian J Pharm Clin Res 2018;11:5-8.

5. Sheth KV, Patel TK, Tripathi CB. Antibiotic sensitivity pattern in neonatal intensive care unit of a tertiary care hospital of India. Asian J Pharm Clin Res 2012;5:46-50.

6. Ahmed A, Lutfi S, Hail MA, Sadi MA. Antibiotic susceptibility patterns of microbial isolates from blood culture in the neonatal intensive care unit of Hamad medical corporation (HMC), Doha, Qatar. Asian J Pharm Clin Res 2013;6:191-5.

7. Mangunkusumo C. National Hospital the Clinical Practice Guidelines of the Department of Pediatrics. Jakarta: National Hospital; 2015.

8. Royal Women's Hospital. Neonatal Pharmacopoeia. $2^{\text {nd }}$ ed. Melbourne: Pharmacy Department the Royal Women's Hospital; 2013.

9. Shann F, editor. Drug Doses. $16^{\text {th }}$ ed. Victoria, Australia: Royal Children's Hospital Parkville; 2014.

10. Satari HI, Firmansyah A, Theresia T, Qualitative evaluation of antibiotic usage in pediatric patients. Pediatr Indones 2011;51:303-10.

11. Pamela DS. Qualitative Evaluation of Antibiotics Usage with Gyssens Method in Class 3 Infection Ward, Departement of Child Health, Dr. Cipto Mangunkusumo Hospital, Prospectively. Thesis. Jakarta: Universitas Indonesia; 2011. 